\title{
Recálculo de las tendencias de mortalidad por accidentes, suicidios y homicidios en Argentina, 1997-2018
}

\author{
Adrián Santoro ${ }^{1}$
}

Forma de citar

Santoro A. Recálculo de lastendencias de mortalidad por accidentes, suicidiosy homicidios en Argentina, 1997-2018. Rev Panam Salud Publica. 2020;44:e74. https://doi.org/10.26633/RPSP.2020.74

RESUMEN

Objetivo. Este estudio se propuso describir el impacto del registro deficiente de la causa de muerte en las tendencias de mortalidad por suicidios y homicidios mediante la imputación de información faltante.

Métodos. Estudio observacional y descriptivo de la tendencia temporal de la mortalidad por causas externas registradas en Argentina en el período 1997-2018. Para la imputación de la intencionalidad, se ajustaron modelos logísticos a partir de variables predictoras provenientes del Informe Estadístico de Defunción. Se utilizaron estadísticas vitales y proyecciones de población como fuentes secundarias.

Resultados. Medida con los datos originales, la mortalidad por causas externas descendió en homicidios y accidentes. Dichos descensos se hicieron más marcados con la información corregida, ya que la imputación impactó con mayor fuerza al principio del período. La mortalidad por suicidios registró un aumento de 8,0\%, que luego de la corrección ascendió a 12,9\%.

Conclusiones. La corrección no modifica de manera sustantiva la tendencia de la mortalidad por causas externas, aunque tiene mayor impacto en la mortalidad por homicidios y suicidios, elevándola.

Palabras clave Registros de mortalidad; violencia; causas de muerte; causas externas; estadísticas vitales; Argentina.

En su "Informe Mundial sobre la Violencia y la Salud", la Organización Mundial de la Salud advirtió sobre la magnitud de la mortalidad por violencias. El aumento de la violencia interpersonal y autoinfligida se plasma en tasas altas de homicidios y suicidios, respectivamente. La tendencia de las tasas de homicidios es a la suba en la Región de las Américas, sobre todo entre los varones, los jóvenes y en los países no industrializados (1).

La mortalidad por causas externas representa un problema de salud pública en Argentina. En el año 2018, murieron aproximadamente 44 habitantes por cada 100000 debido a lesiones, lo que convertía a este grupo de causas en el cuarto en importancia en el país (luego de las enfermedades del sistema circulatorio, los tumores y las enfermedades del sistema respiratorio). Las defunciones por causas externas representaron aproximadamente 20000 muertes al año en las últimas dos décadas (2).
La clasificación de las lesiones con base en la intencionalidad contempla cinco categorías, definidas en función de las circunstancias en las cuales ocurrieron las defunciones. Para este estudio, se focalizó la atención en las agresiones (u homicidios), las lesiones autoinfligidas (o suicidios), los accidentes y los eventos de intención no determinada (EIND). No se tuvieron en cuenta las defunciones debidas a intervenciones legales, por considerarse que responden a determinaciones diferentes a las analizadas.

Argentina presenta algunos déficits en la certificación de las causas de muerte. El uso de categorías poco útiles (3) alcanza a tres de cada diez defunciones (4). Murray y López advirtieron la necesidad de corregir la información de causas de muerte, cuando el registro es deficitario (3).

Los EIND son aquellas defunciones donde la descripción obrante en el Informe Estadístico de Defunción (IED) no es

\footnotetext{
1 Instituto de Salud Colectiva, Universidad Nacional de Lanús, Argentina.

\ Adrián Santoro, adrian.santoro@gmail.com
}

Este es un artículo de acceso abierto distribuido bajo los términos de la licencia Creative Commons Attribution-NonCommercial-NoDerivs 3.0 IGO, que permite su uso, distribución y reproducción en cualquier medio, siempre que el trabajo original se cite de la manera adecuada. No se permiten modificaciones a los artículos ni su uso comercial. Al reproducir un artículo no debe haber ningún indicio de que la OPS o el artículo avalan a una organización o un producto específico. El uso del logo de la OPS no está permitido. Esta leyenda debe conservarse, junto con la URL original del artículo. 
suficiente para clasificarlas dentro de una intencionalidad específica. Esta situación suele surgir de una deficiencia en el registro estadístico de la causa básica. El origen de estos problemas de registro es multicausal y ha sido estudiado en reiteradas oportunidades (5-7).

Para este estudio, se retomó la hipótesis de Zunino, Spinelli y Alazraqui acerca de que los EIND ocultan sobre todo defunciones intencionales (homicidios y suicidios). Los autores denominaron a esta circunstancia como un "eclipse" en los sistemas de información de salud asociado a "una precaria formación de los profesionales a cargo de los registros de mortalidad, la dinámica de los sistemas de información, y la desvalorización de los documentos de registro en las instituciones públicas" (8). Así, las deficiencias en la certificación de la causa de muerte introducirían un sesgo en el análisis de la evolución en el tiempo del fenómeno. El recálculo de la serie a partir de la imputación de los datos faltantes podría componer una tendencia temporal diferente de la descrita con los datos originales, con mayor impacto en los indicadores de mortalidad intencional. Si bien se trata de un problema poco explorado, algunos estudios evidenciaron este fenómeno a través de distintas técnicas para recuperar la información faltante $(9,10)$.

Dilucidar la intencionalidad de esas defunciones impactaría en indicadores sensibles como las tasas de mortalidad por suicidios, homicidios y accidentes, y mejoraría su validez e integridad (11).

En este marco se propuso como objetivo del estudio describir la evolución de la mortalidad por causas externas según su intencionalidad y cómo impactó el registro deficiente de la causa de muerte en dicha tendencia a través del subregistro de la intencionalidad en Argentina.

\section{MATERIALES Y MÉTODOS}

Se realizó un estudio observacional descriptivo de la tendencia temporal de la mortalidad por causas externas registradas en Argentina en el período 1997-2018. La población de estudio estuvo determinada por todas las defunciones registradas en el país en el período señalado. El motivo de la selección del período de estudio radicó en que en el año 1997 se implementó en Argentina la Clasificación Estadística Internacional de Enfermedades y Problemas Relacionados con la Salud (CIE-10) (12), la cual estableció las definiciones utilizadas en la actualidad para la clasificación de la intencionalidad de las defunciones. El final del período estuvo determinado por ser, al momento de la investigación, el último disponible.

La información sobre las defunciones provino de las bases de datos suministradas por la Dirección de Estadísticas e Información del Ministerio de Salud de Argentina. El soporte para la captación de los datos de defunciones se denomina "Informe estadístico de defunción" (IED) y es completado por el médico o médica que registra una defunción. En el caso particular de las defunciones por causas externas, el IED es completado por médicos o médicas forenses, dependientes del Poder Judicial y no del sistema de salud.

Para los denominadores poblacionales, se utilizaron las proyecciones que elabora el Instituto Nacional de Estadística y Censos (INDEC). Debe aclararse que no se dispuso de una serie de proyecciones de población por jurisdicción, grupos de edad y sexo que abarcara todo el período considerado en esta investigación, de manera que se empalmaron dos series de proyecciones diferentes, las provenientes de las series $30,31 \mathrm{y}$ 36 (13-15).

Para el análisis temporal, se calcularon tasas de mortalidad ajustadas por edad (TMAPE) (16). Los intervalos de confianza se calcularon mediante la aproximación normal. Como población estándar se seleccionó la de Argentina según el Censo Nacional de Población, Hogares y Viviendas 2010 (15).

Para la imputación de los datos faltantes se ajustó un modelo logístico donde se utilizaron las siguientes variables independientes provenientes del IED: causa de defunción descripta a través del código de 4 dígitos de la CIE-10 (12), edad agrupada en años de los fallecidos (menos de 5, 5 a 14, 15 a 19, 20 a 24,25 a 29,30 a 49, 50 a 69, 70 y más), sexo de los fallecidos, región de residencia del fallecido (Noreste, Noroeste, Centro, Cuyo y Patagonia) y mecanismo de la defunción (según lista de tabulación para la mortalidad por causas externas utilizada en Argentina) (2). Se reprodujo la metodología de un estudio previo (17). Para la variable independiente se definieron tres categorías: accidentes, homicidios y suicidios, agrupadas según códigos de la CIE-10 (12): accidentes (V01-X59), suicidios (X60-X84; Y870), agresiones (X85-X99; Y00-Y09) y eventos de intención no determinada (Y10-Y34; Y872).

Se imputaron las faltantes a la intencionalidad que obtuvieran mayor probabilidad para cada caso tomando a la categoría “agresión" como basal, según la siguiente ecuación:

$$
p_{i(\text { suicidio/accidente })}=\operatorname{Logit}^{-1}\left(\beta_{0}+\beta_{\text {sexo }_{i}}+\beta_{\text {edad }_{i}}+\beta_{\text {región }_{i}}+\beta_{\text {mecanismo }_{i}}\right)
$$

El método utilizado pudo imputar 43073 EIND (64,0\%). El resto no pudo ser imputado debido a faltantes en las variables predictoras. De esta manera, el porcentaje de EIND sobre el total de las muertes por causas externas se redujo de $15,6 \%$ a $5,6 \%$.

Para todos los años en los que se aplicó el modelo, el estadístico seudo- $\mathrm{R}^{2}$ de Nagelkerke fue mayor a 0,7 y los niveles de significación estadística de las pruebas de verosimilitud menores a 0,05 . Por otro lado, contrastada la eficacia de los modelos para estimar la intencionalidad en los registros que la tenían correctamente asignada, este método arribó a 93,2\% de aciertos en los accidentes, 76,5\% en los suicidios y $84,8 \%$ de los homicidios. A nivel general, las coincidencias alcanzaron $84,8 \%$.

Para el procesamiento y análisis de los datos se utilizaron los siguientes softwares: Microsoft Access $2007^{\circledR}$, Microsoft Excel $2007^{\circledR}$ e IBM SPSS Statistics versión $24^{\circledR}$.

\section{RESULTADOS}

En el período 1997-2018 la tendencia de la mortalidad por causas externas en Argentina fue descendente, aunque la baja no fue constante. Murieron entre 43 y 55 personas por cada 100000 habitantes debido a causas externas según el año observado. Al observar las TMAPE y sus intervalos de confianza, se constató que los años 2003, 2004, 2009, 2014, 2015 y 2016 presentaron descensos significativos con respecto al año inmediato anterior. El año 2011 fue el único del período en el que se registró un alza significativa (figura 1). Los años no mencionados presentaron variaciones no significativas con respecto al año previo.

El porcentaje de EIND fue variable según el grupo etario observado y osciló entre 9,9\% para la población de 80 años y más y 18,0\% para la de 25 a 29 años. Los grupos de edad que concentraron los porcentajes más altos de eventos de intención no determinada fueron los mismos que concentraron los mayores 
FIGURA 1. Tasas de mortalidad por causas externas ajustadas por edad por cada 100000 habitantes, serie original, Argentina, 1997-2018



Elaboración propia en base a datos suministrados por la Dirección de Estadísticas e Información de Salud (Ministerio de Salud de la Nación) y el Instituto Nacional de Estadística y Censos de Argentina.

porcentajes de defunciones por agresiones (todos aquellos que se encuentran entre los 15 y los 34 años). En relación con el sexo, la falta de especificación de la intencionalidad fue algo superior entre los varones $(16,5 \%)$ que entre las mujeres $(12,7 \%)$

La mortalidad por causas externas fue bastante más alta entre los varones. Ese fenómeno se plasma en las tasas de mortalidad a través de altos niveles de desigualdad entre los indicadores de cada sexo. La población de varones comenzó el período con una TMAPE por causas externas de 86,7 por cada 100000 habitantes (intervalo de confianza de 95\% [IC 95\%]: 85,2-88,2), mientras que en las mujeres este indicador presentó un valor de 26,8 (IC95\%: 26,1-27,6) en el año 1997. La mortalidad en varones fue de entre 3,2 y 3,6 veces la de las mujeres en todo el período.

La imputación probabilística de los EIND a alguna de las intencionalidades seleccionadas (accidente, agresión o lesión autoinfligida) permitió estimar un nivel de subestimación para cada intencionalidad y recalcular las TMAPE con el fin de mejorar la descripción de la tendencia. En este análisis se denominó "impacto de la imputación" al porcentaje de variación que experimentó cada TMAPE a partir del recálculo a través de la incorporación de los datos corregidos por la imputación, de manera que aquellos casos donde ese porcentaje fue mayor, más grande fue el impacto.

El modelo de imputación determinó que el nivel de subestimación impactó en primer lugar sobre la TMAPE de homicidios, ya que $28,5 \%$ de los eventos fueron imputados a agresiones. Luego, 19,2\% fueron asignados a lesiones autoinfligidas y $16,5 \%$ a lesiones no intencionales. Entre las muertes de intención no determinada, $36,0 \%$ no pudo ser imputado. En la figura 2 se observa el impacto de la imputación para cada intencionalidad.

Se observó que, luego de asignar la intencionalidad en los EIND, la TMAPE por accidentes aumentó entre 0,7 y 1,9 puntos según el año descrito. En el año 2011, el de mayor impacto de la imputación, la TMAPE resultó un 7,4\% mayor que la original, ya que ascendió de 26,2 por cada 100000 (IC95\%: 25,7-26,7) a 28,1 por cada 100000 (IC95\%: 27,6-28,6). En términos relativos, el impacto de la imputación fue mayor entre las defunciones accidentales de mujeres en comparación con las de varones. El porcentaje de aumento de la TMAPE a partir de la imputación varió entre 2,4\% y $6,9 \%$ en los varones y entre $3,1 \%$ y $9,6 \%$ en las mujeres según el año descrito. El valor mediano del impacto en el período fue $4,7 \%$ en varones y $4,9 \%$ en mujeres. Si bien la tendencia del impacto es hacia una baja leve, sobre el final del período experimentó un repunte, para terminar en 2016 con 5,6\% en varones y 7,9\% en mujeres. El impacto de la imputación para los accidentes no representa un cambio significativo en la tendencia de la mortalidad descrita a partir de las TMAPE sin imputar (figura 3).

En comparación con la mortalidad accidental, la mortalidad por agresiones sufrió en mayor medida el impacto de la imputación de la información faltante (medido como porcentaje de las tasas originales). El aumento que experimentaron las TMAPE nacionales por agresiones a lo largo del período debido a la imputación osciló entre 19,2\% y 57,8\%. En términos absolutos, las variaciones que sufrieron se ubicaron entre 1,1 y 3,6 puntos. Debe destacarse que, en los años 2004, 2005 y 2006, el impacto fue sensiblemente más bajo del valor mediano del período $(45,8 \%)$, de $19,2 \%$, 24,1\% y $24,8 \%$, respectivamente. Descrito 
FIGURA 2. Porcentaje de defunciones por causas externas según intencionalidad: datos originales y datos corregidos por imputación de eventos de intención no determinada, Argentina, 1997-2018

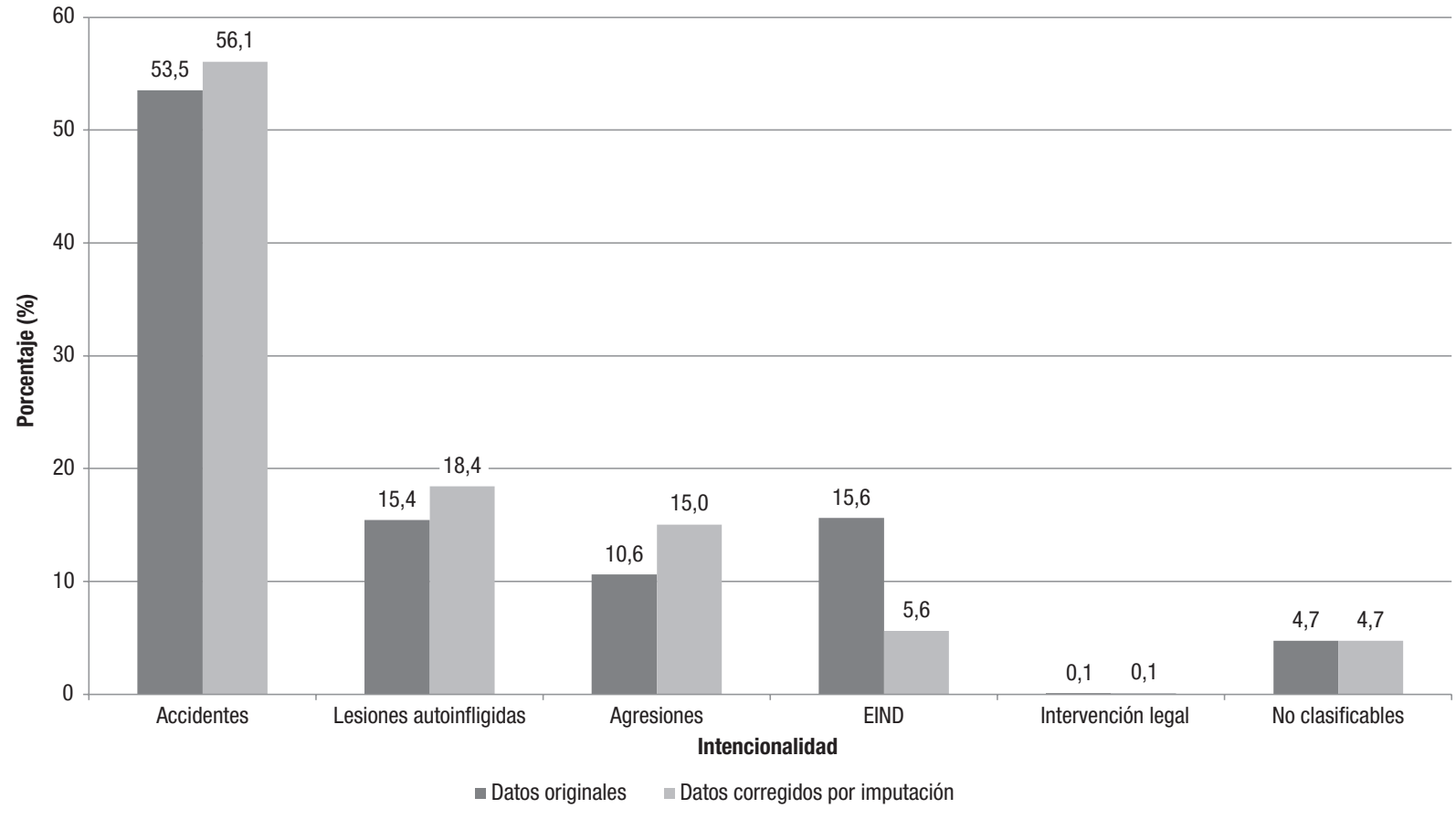

EIND, eventos de intención no determinada.

Elaboración propia en base a datos suministrados por la Dirección de Estadísticas e Información de Salud (Ministerio de Salud de la Nación) y el Instituto Nacional de Estadística y Censos de Argentina.

FIGURA 3. Tasas de mortalidad por accidentes ajustadas por cada 100000 habitantes: serie original y serie corregida, Argentina, 1997-2018

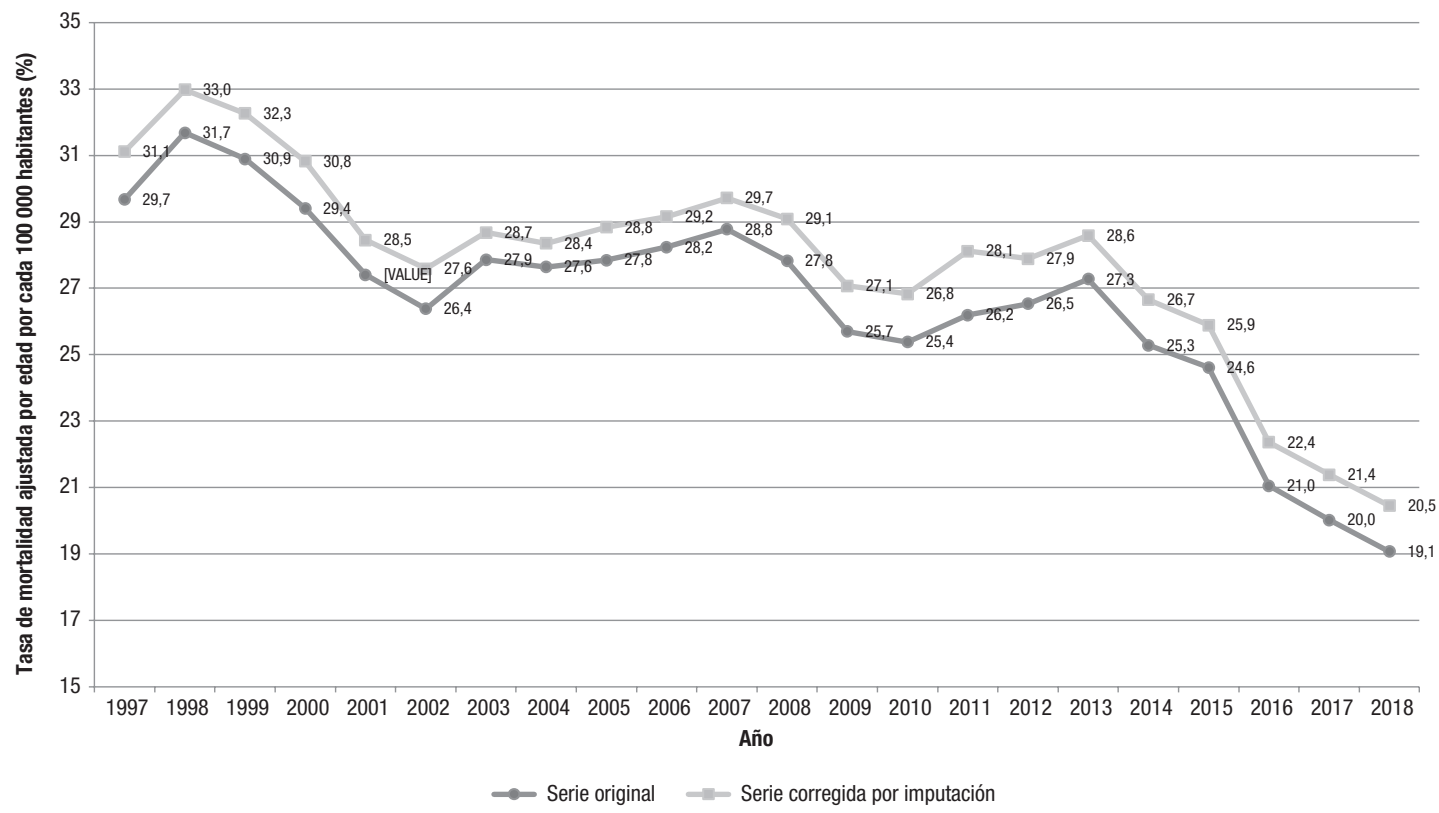

Elaboración propia en base a datos suministrados por la Dirección de Estadisticas e Información de Salud (Ministerio de Salud de la Nación) y el Instituto Nacional de Estadística y Censos de Argentina. 
FIGURA 4. Tasas de mortalidad ajustadas por edad por causas externas intencionales por cada 100000 habitantes: serie original y serie corregida, Argentina, 1997-2018

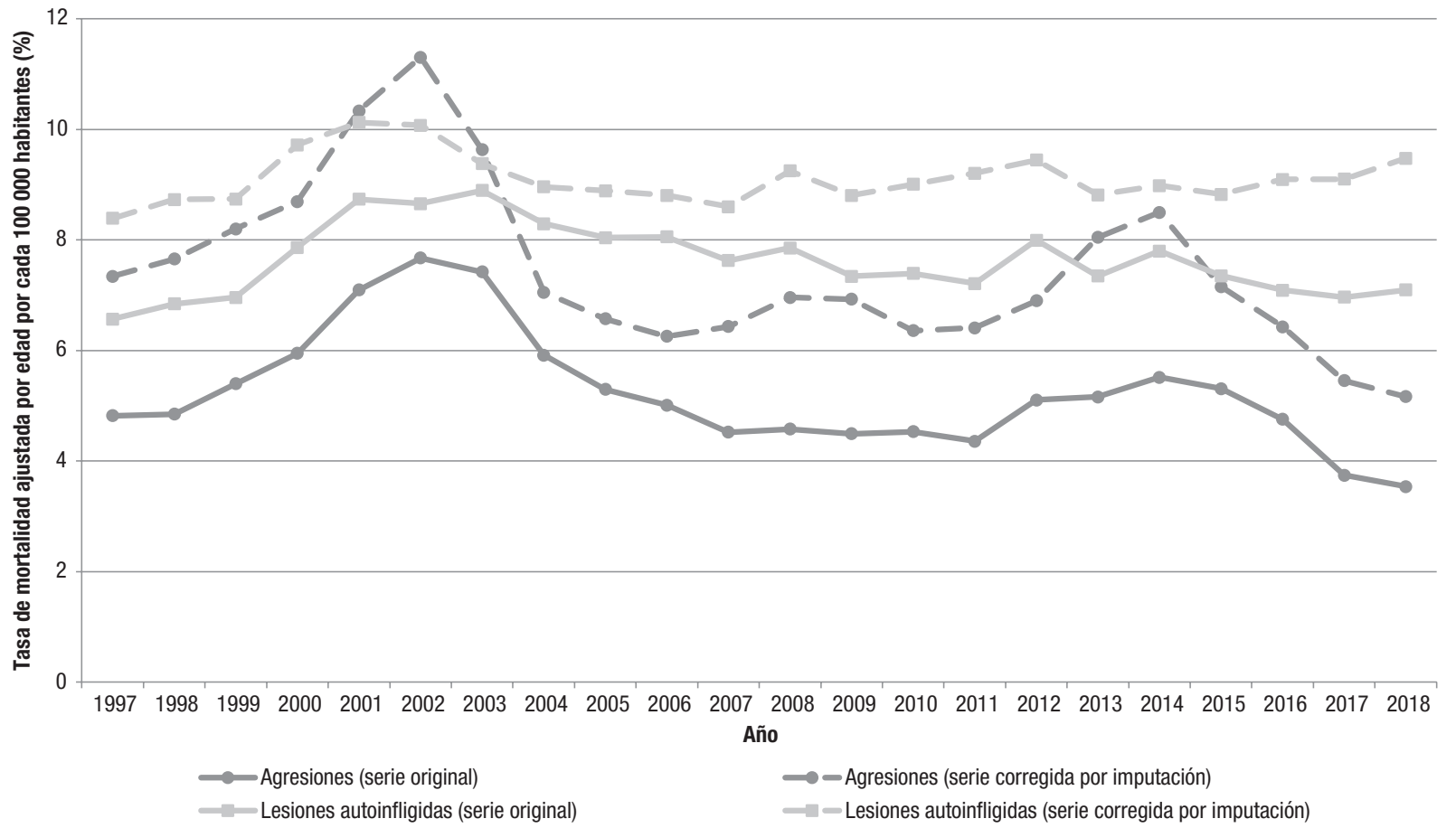

Elaboración propia en base a datos suministrados por la Dirección de Estadísticas e Información de Salud (Ministerio de Salud de la Nación) y el Instituto Nacional de Estadística y Censos de Argentina.

por sexo, se observó que en la mortalidad accidental el valor mediano de aumento de la mortalidad del período en varones fue bastante superior al de mujeres: $47,5 \%$ versus $33,4 \%$, respectivamente. Debe destacarse que, para el año 1998, el impacto de la imputación de la TMAPE por agresiones en varones alcanzó $60,3 \%$, que se constituyó como el valor máximo. Es importante resaltar que, como resultado de la imputación, la mortalidad por agresiones en Argentina en el período estudiado evidenció un descenso más marcado que el descrito mediante los datos sin imputar, debido a que el nivel de subenumeración de homicidios al principio del período fue mayor al del final. De todas formas, y como consecuencia del mismo factor, el nivel de la mortalidad al final del período fue de $45,9 \%$ más alto que el registrado con los datos sin imputar.

Para todos los años incluidos en el análisis el porcentaje de aumento debido a la imputación de la intencionalidad a la TMAPE por suicidios estuvo entre 5,4\% y 33,6\%. Resulta interesante observar que, a partir de las TMAPE imputadas, la serie adquiere una tendencia donde se concentran los niveles más altos de mortalidad entre los años 2001 y 2003. Por otro lado, como consecuencia de que el impacto de la imputación se acrecienta a medida se avanza en la cronología, el incremento de la mortalidad por suicidios fue mayor entre el principio y el fin del período (en términos absolutos y de variación relativa) en comparación con la serie no corregida ( $8,0 \%$ en la serie original y $12,9 \%$ en la imputada). La TMAPE por suicidios en el año 2018 aumenta un tercio al imputar los EIND, y alcanza el valor de 9,5 por cada 100000 habitantes
(IC95\%: 9,2-9,8). Por su parte, con los datos originales se ubica en 7,1 por cada 100000 habitantes (IC95\%: 6,8-7,3). Al comparar entre varones y mujeres, la imputación no aportó diferencias sustanciales. Se mantuvo la misma tendencia de la información original: TMAPE más altas y a la suba en varones, y en leve descenso para las mujeres. De todos modos, pasan a ser más altas en varones y mujeres debido a la recuperación de defunciones a través del proceso de imputación. Se mantienen, además, los años del principio de la década del 2000 como los momentos de mayor nivel de mortalidad por lesiones autoinfligidas.

En la figura 4 pueden apreciarse las tendencias originales y corregidas de muertes por causas intencionales (agresiones y lesiones autoinfligidas).

\section{DISCUSIÓN}

El estudio realizado ha puesto en evidencia algunos elementos relevantes para la descripción del fenómeno de la mortalidad por causas externas en Argentina en la historia reciente. En términos epidemiológicos, un hallazgo para destacar es que la mortalidad por suicidios ha aumentado en los últimos 20 años, en un contexto en el que el resto de las intencionalidades han disminuido y que, al imputar la información faltante, el aumento es aún mayor. Este hallazgo debe evaluarse con precaución debido a que el modelo logístico mostró menor eficacia en la asignación de suicidios. Acerca de ello, podría hipotetizarse que debido a que las distribuciones de edad y 
FIGURA 5. Cantidad de defunciones por agresiones, serie original (Dirección de Estadística e Información de Salud, DEIS), serie corregida por imputación (DEIS) y serie del Sistema Nacional de Información Criminal, Argentina, 2001-2018

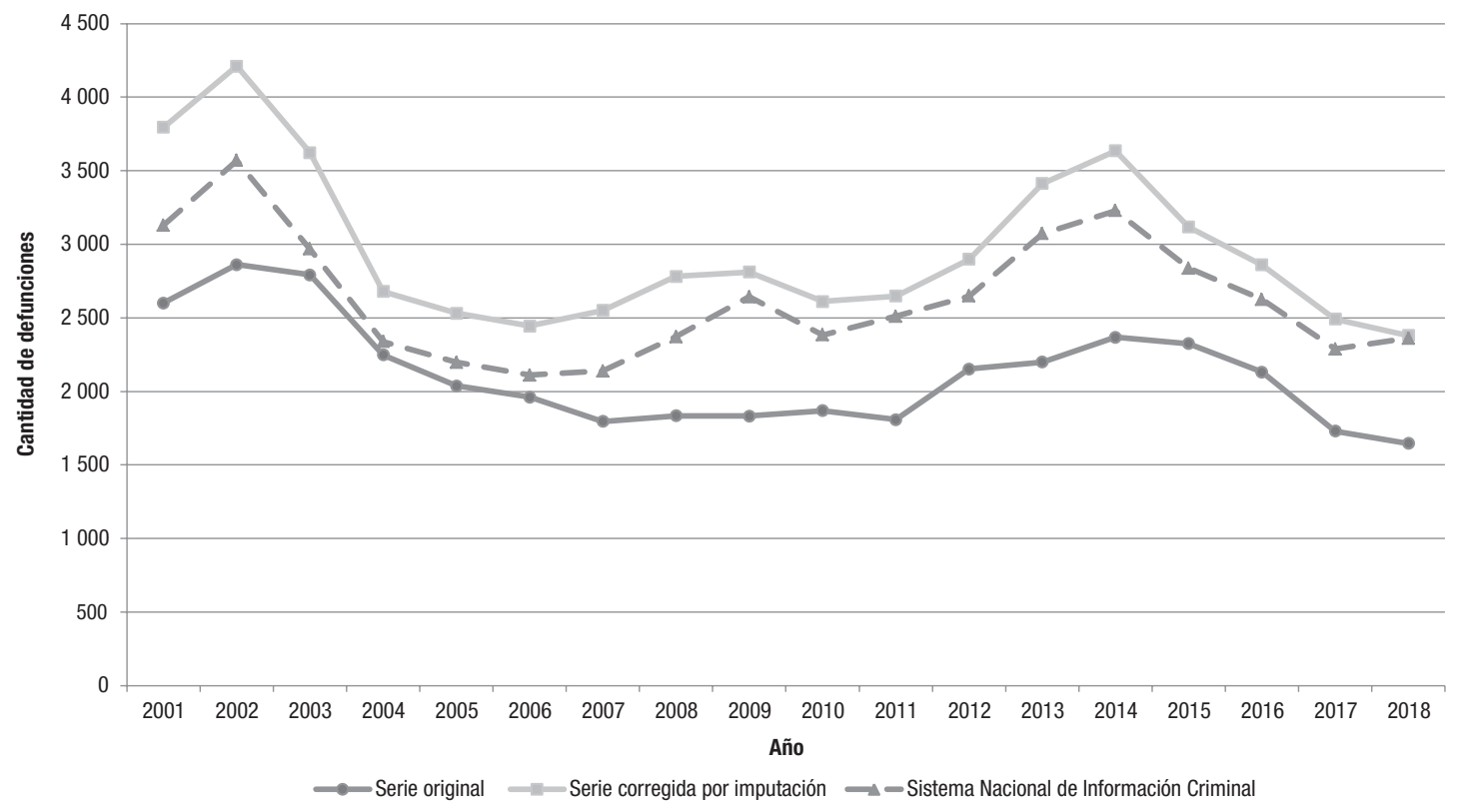

DEIS, Dirección de Estadísticas en Información en Salud; SNIC, Sistema Nacional de Información Criminal.

Elaboración propia en base a datos suministrados por la Dirección de Estadísticas e Información de Salud y el Sistema Nacional de Información Criminal de Argentina.

sexo en la mortalidad intencional son similares para suicidios y homicidios (en tanto las violencias ocurren de manera más frecuentes en adultos jóvenes), resulta más complejo predecir la intencionalidad.

En términos metodológicos, el modelo logístico imputó la intencionalidad de manera no proporcional a la distribución de las defunciones con intencionalidad registrada, lo que confirma que la distribución de este déficit en el llenado de los registros estadísticos no es aleatorio; se confirma parcialmente así la hipótesis planteada oportunamente por Zunino, Spinelli y Alazraqui $(8)$ y observada en otros estudios $(9,10)$ ya que si bien el impacto de la imputación se carga sobre todo sobre las muertes intencionales, dicha carga no modifica de manera sustantiva la tendencia temporal.

Andreev (10) y otros habían obtenido resultados semejantes en la aplicación de modelos logísticos a la imputación de EIND, concentrándose en las categorías intencionales los eventos imputados. Sin embargo, pudieron observarse algunas diferencias: en el estudio citado hubo menos imputaciones, en términos relativos, hacia los homicidios (19,5\% de los EIND frente a $28,5 \%$ en el presente estudio). Para el caso de los suicidios, se obtuvieron resultados muy similares en ambos estudios: $21,4 \%$ en el estudio citado y $19,2 \%$ en el presente. Estudios basados en metodologías de pesquisa de otras fuentes de información también arribaron a mayores aportes de la imputación hacia las categorías intencionales $(9,18)$.

Las investigaciones de Santoro (17) y Adreev et al. (10) representan los únicos antecedentes encontrados de aplicación de modelos logísticos a la imputación de EIND; sin embargo, el segundo presenta una alternativa metodológica para imputar la totalidad de los eventos.
Resulta interesante observar el acercamiento que se produce en la serie de defunciones por agresiones con los datos corregidos mediante la imputación y los que provienen del Sistema Nacional de Información Criminal (SNIC), que registra las muertes por "homicidios dolosos" desde 2001 (19). Se observó que la brecha entre ambas series es irregular, de manera que en futuros estudios debieran evaluarse otras variables de ambos sistemas que podrían intervenir en ese resultado (figura 5). Queda planteado también el interrogante acerca de las causas que determinan que la serie imputada supere a la de homicidios dolosos del SNIC, donde podrían estar interviniendo deficiencias del modelo de imputación de este estudio o el subregistro de homicidios dolosos por parte del otro sistema. Otros elementos para analizar en profundidad son las definiciones conceptuales de ambas mediciones, debido a que con frecuencia se asume la equivalencia entre los "homicidios dolosos" del Sistema Criminal y las "agresiones" del Sistema Estadístico de Salud.

Los resultados mostraron un impacto considerable de la imputación, lo que permite enfatizar la necesidad de buscar alternativas para resolverlo en pos de lograr una mejora en la calidad de indicadores estratégicos para el diseño y la implementación de políticas públicas para la prevención de las violencias.

La falta de información registrada en las variables predictoras no ha permitido imputar la intencionalidad para la totalidad de los hechos registrados de forma incompleta, lo que representa una debilidad de este estudio. En ese sentido, resulta importante destacar que dicha falta se concentra principalmente en la información sobre el mecanismo que produjo la muerte (dato faltante en $22,6 \%$ de las muertes analizadas) mientras que, en el sexo, la edad y la jurisdicción de residencia, existe un nivel muy bajo de omisión (no supera el 1,0\%). Puede constituir otra debilidad 
no haber profundizado al interior de cada intencionalidad, por lo que no se han diferenciado, por ejemplo, los suicidios según su mecanismo ni identificado las muertes por armas de fuego. Además, el método de imputación utilizado es probabilístico y, como es natural, vulnerable al error de predicción. Otra limitación del estudio deviene de que las determinaciones de la mortalidad por causas externas y del no registro de la intencionalidad pueden estar asociadas a cuestiones vinculadas al entorno habitual del fallecido o de la fallecida $\mathrm{y}$, a su vez, con las circunstancias de ocurrencia del hecho, por lo cual optar por un análisis basado en el área geográfica de residencia como en el de ocurrencia representa potencialmente un sesgo. La inexistencia de una serie única de proyecciones de población para todo el período estudiado constituye también una limitación.

Por otra parte, este estudio posee la fortaleza de reconstruir una serie estadística con menor pérdida de información de la serie original. Estudios previos habían evidenciado las dificultades que implica a la investigación sobre muertes violentas el déficit en la certificación de la causa de muerte en Argentina $(20,21)$. En términos epidemiológicos, permite aportar mayor conocimiento a la dinámica de la mortalidad por causas externas y a su nivel actual.

Puede concluirse que las tendencias de la mortalidad por causas externas no se alteran mediante la imputación de los datos faltantes, pero sí experimentan incrementos heterogéneos, donde la mortalidad por suicidios se ve incrementada. Por otro lado, que la técnica utilizada para la imputación proviene de información del mismo registro de la defunción, lo que, desde el punto de vista cualitativo, es superador de los métodos de distribución de las defunciones inespecíficas que se basan en criterios provenientes de agregados estadísticos (por proporcionalidad y distribuciones a priori, entre otros). Como se mencionó antes, el estudio de los EIND se halla aún en fase inicial, con pocos antecedentes de estudio, lo que hace necesaria la profundización de este trabajo para lograr una metodología validada y generalizable.

En función de las características del Sistema Estadístico de Salud de la Argentina y, en relación con la magnitud del problema, resulta necesario rever la forma de captación de la información estadística de causa de muerte por lesiones. Palomo Rando et al. (7) detectaron que intervienen polisemias y diferencias conceptuales entre las disciplinas que intervienen en el registro médico y legal que podrían actuar en desmedro de la calidad de la información. En Argentina, no solo las defunciones por causas externas presentan problemas de calidad en la certificación. Se han constatado también altos niveles de causas "mal definidas" $\mathrm{y}$, sobre todo, "poco útiles" dentro del resto de las muertes (4, 22). En ese contexto, la modernización del Sistema de Estadísticas Vitales debiera considerarse como prioridad: el pasaje al registro electrónico de las defunciones, la unificación de registros legales y estadísticos, la implementación de sistemas codificación automatizada y el funcionamiento permanente de un ámbito institucional que concentre a todos los actores involucrados en la producción de información de mortalidad posibilitaría el mejoramiento de la calidad de la información estadística.

Contribución del autor. AS concibió el estudio original, analizó los datos, interpretó los resultados, escribió y revisó el manuscrito.

\section{Conflictos de intereses. Ninguno declarado por el autor.}

Financiación. El estudio representa una actualización del estudio inédito "Descripción de la tendencia temporal de la mortalidad por causas externas según intencionalidad en Argentina a partir de la imputación de los eventos de intención no determinada (1997-2016)" desarrollado durante los años 2018 y 2019 bajo el programa de becas individuales de la Dirección de Investigación para la Salud del Ministerio de Salud de la Nación de Argentina. Los patrocinadores no participaron de ninguna manera en el diseño del estudio, la colecta y análisis de los datos, la decisión de publicar este trabajo ni la preparación del manuscrito.

Declaración. Las opiniones expresadas en este manuscrito son únicamente responsabilidad del autor y no reflejan necesariamente los de la Revista Panamericana de Salud Pública o la Organización Panamericana de la Salud.

\section{REFERENCIAS}

1. Krug EG, Mercy JA, Dahlberg LL, Zwi AB. El informe mundial sobre la violencia y la salud. Biomédica. 2002;22:327-36.

2. Ministerio de Salud de la Nación Argentina, Dirección de Estadísticas e Información de Salud. Estadísticas Vitales. Información Básica 2019. Ciudad Autónoma de Buenos Aires: Ministerio de Salud de la Nación, Dirección de Estadísticas e Información de Salud; 2019.

3. Murray CL, Lopez AD. The Global Burden of Disease. Ginebra: Organización Mundial de la Salud; 1996.

4. Ministerio de Salud de la Nación Argentina, Dirección de Estadísticas e Información de Salud. Reporte Interactivo de Estadísticas de Salud, 2020. Disponible en: : http://deis.msal.gov.ar/ReporteInteractivo/ Acceso el 18 de abril de 2020.

5. de Arán Barés M, Pérez G, Rosell J, Molina P. Exactitud de las estadísticas de mortalidad por causas externas y naturales con intervención médico-legal en Cataluña, 1996. Gac Sanit. 2000;14(5):356-362.

6. Schottenfeld D, Muzza Eaton MD, Sommers SC, Alonso DR, C W. The autopsy as a measure of accuracy of the death certificate. Bull NY Acad Med. 1982;58(9):778-94.

7. Palomo Rando JL, Ramos Medina V, Cruz Mera E, Calvo, López AM. Diagnóstico del origen y la causa de la muerte después de la autopsia médico-legal (Parte II). Cuad Med Forense. 2011;17(1):7-12.
8. Zunino MG, Spinelli H, Alazraqui M. Muertes por armas de fuego: un eclipse en los sistemas de información en salud. Salud Colect. 2006;2(3):259-67.

9. Fontain G, Castelucci J, Rodríguez S, Toledo L, Parolini S, Lescano Pareja J, et al. Reparo de muertes adolescentes por "eventos de intención no determinada o causa mal definida" en Córdoba, 2015. Revista de la Facultad de Ciencias Médicas de Córdoba. 2018:195-6. Disponible en: https://doi.org/10.31053/1853.0605.v0.n0.21254

10. Andreev E, Shkolnikov VM, Pridemore WA, Nikitina SY. A method for reclassifying cause of death in cases categorized as "event of undetermined intent." Popul Health Metr. 2015;13(23). Disponible en: https://doi.org/10.1186/s12963-015-0048-y

11. Organización Panamericana de la Salud. Indicadores de salud: aspectos conceptuales y operativos. Washington D.C.: OPS; 2014.

12. Organización Panamericana de la Salud. Clasificación estadística internacional de enfermedades y problemas relacionados con la salud. Décima revisión (CIE-10). Washington D.C.: OPS; 2003.

13. Instituto Nacional de Estadística y Censos (INDEC). Proyecciones provinciales de población por sexo y grupos de edad 2001-2015. No 31 Serie Análisis Demográfico. Ciudad Autónoma de Buenos Aires: INDEC; 2005. 
14. Instituto Nacional de Estadística y Censos (INDEC). Proyecciones provinciales de población por sexo y grupos de edad 2001-2015. No 31 Serie Análisis Demográfico. Ciudad Autónoma de Buenos Aires: INDEC; 2013.

15. Instituto Nacional de Estadística y Censos (INDEC). Estimaciones y proyecciones de población. Total del país. 1950-2015. № 30 Serie Análisis Demográfico. Ciudad Autónoma de Buenos Aires: INDEC; 2004.

16. Organización Panamericana de la Salud. Lineamientos básicos para el análisis de la mortalidad. Washington D.C.: OPS; 2017.

17. Santoro A. Factores socio-demográficos asociados a la mortalidad por causas externas en Argentina (1999-2003) (tesis de maestría). Universidad Nacional de Tres de Febrero; 2012.

18. Matos SG, Proietti FA, Barata R de CB. Confiabilidade d informação sobre mortalidade por violência em Belo Horizonte, MG. Rev Saude Publica. 2007;41(1):76-84.

19. Ministerio de Seguridad, Secretaría de Gestión Federal de la Seguridad, Subsecretaría de Estadística Criminal, Dirección del Sistema Nacional de Información Criminal. Estadísticas Criminales - Homicidios Dolosos - Serie histórica - 2001 al 2018 [Internet]. Ciudad Autónoma de Buenos Aires: Ministerio de Seguridad; 2019. Disponible en: https://estadisticascriminales.minseg.gob
.ar/reports/Informe serie historica HOMICIDIOS DOLOSOS 2001 al 2018.pdf

20. Escanés G. Evolución de la mortalidad por atropellos y colisiones de tránsito en Argentina entre 2001 y 2010. Rev Bras Estud Popul. 2015;32(1):49-71.

21. Andrada MJ, Bertone CL. Análisis de las defunciones por accidentes de tránsito en los registros oficiales de La Rioja, Argentina (20112013). Mem Inst Investig Cienc Salud. 2017;15(2):6-13. Disponible en: https:/ / ri.conicet.gov.ar/bitstream/handle/11336/75656/ CONICET_Digital_Nro.3caf1e0b-f880-41e7-8375-c1709850560d_A .pdf? sequence $=2 \&$ isAllowed $=y$

22. Ribotta BS. Causas de defunción mal definidas en las provincias de Argentina, 2001-2013. Memorias del Inst Investig en Ciencias la Salud. 2016;14(3).

Manuscrito recibido el 8 de enero de 2020. Aceptado para su publicación, tras revisión, el 23 de abril de 2020

\section{Recalculation of trends in mortality from accidents, suicides, and homicides in Argentina, 1997-2018}

SUMMARY

Keywords
Objective. To describe the impact of deficient cause-of-death records on trends in death by suicide and homicide, through imputation of missing information.

Methods. Observational and descriptive study of temporal trends in recorded deaths from external causes in Argentina in the period 1997-2018. For imputation of intent, logistic models were adjusted on the basis of predictive variables from the Statistical Report on Deaths. Vital statistics and population projections were used as secondary sources.

Results. As measured by the original data, mortality from external causes declined, specifically for homicides and accidents. These declines were more pronounced with the corrected data, since imputation of intent had a greater impact early in the period. Death by suicide increased by $8.0 \%$; and after the correction, by $12.9 \%$. Conclusions. The correction does not substantively alter the trend in mortality from external causes, but it does significantly increase mortality from homicide and suicide.

\section{Recálculo das tendências de mortalidade por acidentes, suicídios e homicídios na Argentina, 1997-2018}

RESUMO Objetivo. Este estudo procurou descrever o impacto do registro inadequado da causa de morte sobre as tendências de mortalidade por suicídio e homicídio, por meio da imputação de informações ausentes.

Métodos. Estudo observacional e descritivo da tendência temporal de mortalidade por causas externas registradas na Argentina no período de 1997 a 2018. Para imputar a intencionalidade, ajustamos os modelos logísticos utilizando variáveis preditivas provenientes do Relatório Estatístico de Óbitos. Utilizamos estatísticas vitais e projeções populacionais como fontes secundárias.

Resultados. Medida com os dados originais, a mortalidade por causas externas diminuiu em termos de homicídios e acidentes. Essa diminuição foi mais acentuada com as informações corrigidas, uma vez que a imputação teve um impacto mais forte no início do período de estudo. A mortalidade por suicídio registrou um aumento de $8,0 \%$, que subiu para $12,9 \%$ após a correção.

Conclusões. A correção não modifica substancialmente a tendência de mortalidade por causas externas, mas tem um maior impacto sobre a mortalidade por homicídios e suicídios, elevando-a.

Palavras-chave Registros de mortalidade; violência; causas de morte; estatísticas vitais; Argentina. 